\title{
Readmissions and costs among younger and older adults for targeted conditions during the enactment of the hospital readmission reduction program
}

\author{
Chi-Hua Lu, Collin M. Clark, Ryan Tober, Meghan Allen, Walter Gibson, Edward M. Bednarczyk,
} Christopher J. Daly and David M. Jacobs ${ }^{*}$ (iD

\begin{abstract}
Background: The Hospital Readmissions Reduction Program (HRRP) was introduced to reduce readmission rates among Medicare beneficiaries, however little is known about readmissions and costs for HRRP-targeted conditions in younger populations. The primary objective of this study was to examine readmission trends and costs for targeted conditions during policy implementation among younger and older adults in the U.S.

Methods: We analyzed the Nationwide Readmission Database from January 2010 to September 2015 in younger (18-64 years) and older ( $\geq 65$ years) patients with acute myocardial infarction (AMI), heart failure (HF), pneumonia, and acute exacerbations of chronic obstructive pulmonary disease (AECOPD). Pre- and post-HRRP periods were defined based on implementation of the policy for each condition. Readmission rates were evaluated using an interrupted time series with difference-in-difference analyses and hospital cost differences between early and late readmissions ( $\leq 30$ vs. $>30$ days) were evaluated using generalized linear models.

Results: Overall, this study included $16,884,612$ hospitalizations with $3,337,266$ readmissions among all age groups and 5,977,177 hospitalizations with 1,104,940 readmissions in those aged 18-64 years. Readmission rates decreased in all conditions. In the HRRP announcement period, readmissions declined significantly for those aged 40-64 years for AMI $(p<0.0001)$ and HF ( $p=0.003)$. Readmissions decreased significantly in the post-HRRP period for those aged $40-64$ years at a slower rate for AMI $(p=0.003)$ and HF $(p=0.05)$. Readmission rates among younger patients (1864 years) varied within all four targeted conditions in HRRP announcement and post-HRRP periods. Adjusted models showed a significantly higher readmission cost in those readmitted within 30 days among younger and older populations for AMI ( $p<0.0001)$, HF ( $p<0.0001)$, pneumonia $(p<0.0001)$, and AECOPD $(p<0.0001)$.

Conclusion: Readmissions for targeted conditions decreased in the U.S. during the enactment of the HRRP policy and younger age groups (<65 years) not targeted by the policy saw a mixed effect. Healthcare expenditures in younger and older populations were significantly higher for early readmissions with all targeted conditions. Further research is necessary evaluating total healthcare utilization including emergency department visits, observation units, and hospital readmissions in order to better understand the extent of the HRRP on U.S. healthcare.
\end{abstract}

Keywords: Readmissions, NRD, Younger adults, HRRP, Targeted conditions, Costs

\footnotetext{
* Correspondence: dmjacobs@buffalo.edu
}

Department of Pharmacy Practice, School of Pharmacy and Pharmaceutical

Sciences, University at Buffalo, 316 Pharmacy Building, Buffalo, NY, USA

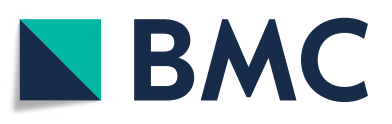

(๑) The Author(s). 2021 Open Access This article is licensed under a Creative Commons Attribution 4.0 International License, which permits use, sharing, adaptation, distribution and reproduction in any medium or format, as long as you give appropriate credit to the original author(s) and the source, provide a link to the Creative Commons licence, and indicate if changes were made. The images or other third party material in this article are included in the article's Creative Commons licence, unless indicated otherwise in a credit line to the material. If material is not included in the article's Creative Commons licence and your intended use is not permitted by statutory regulation or exceeds the permitted use, you will need to obtain permission directly from the copyright holder. To view a copy of this licence, visit http://creativecommons.org/licenses/by/4.0/. The Creative Commons Public Domain Dedication waiver (http://creativecommons.org/publicdomain/zero/1.0/) applies to the data made available in this article, unless otherwise stated in a credit line to the data. 


\section{Background}

Reducing avoidable and costly re-hospitalizations within 30 days of discharge among Medicare beneficiaries in the United States has become a consensus from governments and researchers [1]. The Hospital Readmissions Reduction Program (HRRP) was initially introduced as part of the Patient Protection and Affordable Care Act (PPACA) in 2010 with the goal of creating financial in-

centives to reduce readmission rates and improve transitional care among Medicare beneficiaries [2]. In October 2012, hospitals began incurring financial penalties if 30day, all-cause, risk-standardized readmission rates were higher than expected for Medicare beneficiaries with the following conditions: acute myocardial infarction (AMI), heart failure (HF), and pneumonia. In 2014, the HRRP was expanded to target additional conditions including acute exacerbations of chronic obstructive pulmonary disease (AECOPD) [2]. The penalties associated with the HRRP increased to $3 \%$ of all Medicare payments for fiscal year (FY) 2015, and > 2500 hospitals will face HRRP penalties for FY 2020 [3].

Current data show a relationship between the HRRP and a reduction in readmissions for the targeted conditions among Medicare beneficiaries [2-4]. Zuckerman et al. reported a decrease in hospital-level readmission rates for targeted HRRP conditions among elderly Medicare beneficiaries from 2007 to 2015 (21.5 to 17.8\%) [2]. Less is known about readmission trends for the targeted conditions in younger, non-Medicare populations, in whom these conditions are increasingly common. Readmissions in younger populations covered by Medicaid or private insurance account for a high percentage of all readmissions (21 and 12\%, respectively), with estimated total costs of over \$1.5 billion [4-6]. Previous studies on readmissions among younger populations have not included AECOPD, a major condition targeted by the HRRP, and are limited to select states and years $[4,7,8]$. Broader knowledge of readmissions and costs for the four major targeted conditions among a nationally representative cohort of younger patients may help tailor clinical initiatives and policy development.

Initiatives are being developed across the health continuum to improve readmission rates through the implementation of transition of care and readmission reduction programs [9-12]. These programs may include younger populations that are not included as part of HRRP policy. Understanding readmission trends and costs for younger and older populations from large database in the context of the HRRPtargeted conditions may incentivize improvements in these transition programs. Therefore, the objectives of this study were to: (i) examine readmission trends for HRRP-targeted conditions including AMI, HF, pneumonia, and AECOPD prior to and after implementation of the policy among younger and older populations in the United States and (ii) evaluate the hospital cost differences between early and late readmission events. This study is novel in the following ways: (i) readmission trends for HRRP targeted conditions were evaluated and stratified based on three different age groups $(18-39,40-64, \geq 65$ years of age)

and (ii) we examined cost differences across the four conditions between early ( $\leq 30$ days) and late $(>30$ days) readmissions within younger and older populations.

\section{Methods}

\section{Data source}

We analyzed data from the Nationwide Readmission Database (NRD) developed by the Agency for Healthcare Research and Quality (AHRQ) for the Healthcare Cost and Utilization Project (HCUP) as it is a largescale administrative database designed specifically to support analyses of national readmissions for all ages and payers [13]. The NRD contains year-specific verified patient linkage numbers that can be used to track persons across hospitals in a state within a calendar year. The database includes all discharge data from community hospitals in 27 states but not from rehabilitation or long-term acute care hospitals. The NRD is designed to generate national estimates of readmissions since it includes over half of the U.S. population and hospitalizations each year. For example, in 2015, the database included 17.2 million raw discharges and 36 million weighted discharges representing $56.6 \%$ of all U.S. hospitalizations. The University at Buffalo Institutional Review Board exempted this study from review, as data were deidentified and publicly available through the AHRQ.

\section{Study population}

We included all adults $\geq 18$ years with an International Classification of Diseases, Ninth Revision, Clinical Modification (ICD-9-CM) code for AMI, HF, and pneumonia between January 2010 and September 2015. For AECOPD, we only included adults aged $\geq 40$ years, as this threshold has been used to help exclude patients with asthma exacerbations [14]. We also identified nontargeted conditions, representing hospitalizations excluding AMI, HF, pneumonia, and AECOPD as our control cohort. The ICD-9-CM codes were chosen based on those published by the Centers for Medicare and Medicaid Services (CMS) for the HRRP for assessment of allcause readmissions (S1, S2, S3-4 Tables) [15-18]. We excluded patients if they died during the index hospitalization, discharged against medical advice, or if 
they were residents of a different state. As recommended by the HCUP, we used data for 11 months (January to November) to capture all 30-day follow-up for all patients from 2010 to 2014. We used data for nine months (January to September) in 2015 since International Classification of Diseases, Tenth Revision, Clinical Modification (ICD-10-CM) codes were implemented on October 1. We included subjects up to September 2015 given the substantial differences between ICD-9-CM to ICD-10$\mathrm{CM}$ codes and lack of comparison of the two coding systems in the U.S.

\section{Patient and hospital covariates}

The NRD classifies covariates as demographic, preexisting comorbidity, clinical, and hospital characteristics. Patient demographic variables included were age, gender, insurance status, and median household income. Age was stratified into $18-39,40-64$, and $\geq 65$ years groups for AMI, HF, pneumonia, and non-targeted conditions. For AECOPD, age was stratified into 40-64 and $\geq 65$ years groups. Insurance status collected by the database was designated as the primary insurance payer for each admission. Insurance was categorized as Medicare, Medicaid, private insurance, self-pay, or other (including no charge). Income levels were based on the estimated median household income of residents in the patient's zip code and were divided into $\$ 1-\$ 41,999$, $\$ 42,000-\$ 51,999, \$ 52,000-\$ 67,999$, and $>\$ 68,000$. Preexisting comorbidities were assessed as overall comorbidity burden using the Elixhauser comorbidities provided by the AHRQ [19]. Discharge disposition was categorized as home, home healthcare, or skilled nursing facility and other (including transfers to immediate care, another type of facility, or discharged alive but destination unknown). Hospitalization characteristics included length of stay in days and hospital charges associated with the index hospitalization and readmission.

\section{Outcomes}

The primary outcome of this study was 30-day all-cause unplanned readmission rate. We followed the CMS methodologies for condition specific readmission measures to construct our analytical cohorts (AMI, HF, pneumonia and AECOPD) for each age group [15-18]. Briefly, the CMS measures only define the first readmission within 30 days of discharge as a 30-day readmission. Additional readmissions within the 30 -day period are not counted as 30-day readmissions or index hospitalizations for the same condition. After 30 days from discharge, hospitalizations are counted as index admissions if they meet the inclusion criteria. The secondary outcome was costs of index hospitalization and readmission. Costs were defined as the amount that hospitals billed for services and were standardized to 2015 dollars using the index from the U.S. Bureau of Labor Statistics [20]. We evaluated differences in readmissions costs by stratifying readmissions as either an early ( $\leq 30$ days) or late readmission ( $>30$ days) and further stratified by age groups including $18-39,40-64$, and $\geq 65$ years. Late readmissions were defined patients re-entered to hospitals above 30 days up to the end of each year due to the nature of NRD.

\section{Statistical analysis}

To account for the complex survey design of the NRD, we used a survey-specific methodology with hospital as clusters, NRD stratum as strata, and discharge-level weights as weights to obtain study population data and weighted nationwide overall and annual 30-day readmission rates for each targeted condition. Demographic and clinical characteristics were compared between the targeted conditions. Categorical variables were reported as percentages and compared using the chi-square test and continuous variables were reported as medians (interquartile ranges, IQR) and compared using the MannWhitney U test.

We first evaluated overall changes including all ages and then stratified the data into three age-specific groups (18-39, 40-64, $\geq 65$ years). Readmission rates were evaluated using a segmented regression analysis of interrupted time series (ITS) data to assess changes in level and slope of the regression lines in the HRRP announcement phase and post-HRRP segments. This analysis allowed the estimation of changes in outcomes between HRRP announcement and post-HRRP phases while accounting for both sudden changes and changes in trends for the outcome of interest. A change in level would indicate an immediate effect of the HRRP on the outcome of interest, while a change in slope (or trend) of the regression line would point to a longer, more sustained response for the outcome measured. Then, we expanded our segmented regression models with the difference-in-difference (DD) approach among younger and older populations separately [21, 22]. We calculated the difference in rates (e.g. the rate of AMI in 18-39 age group minus the rate of non-targeted conditions in 1839 age group for every time period) for each targeted condition [21]. Taking the difference allowed us to collapse the 2 time series into 1 in order to estimate a DD effect and also estimate how the change in readmissions rates of each targeted condition differed from nontargeted conditions over the same time period.

The ITS analysis is a quasi-experimental approach for evaluating longitudinal effects of policy interventions, and the interventions are different for each condition [23, 24]. For AMI, HF, and pneumonia, the HRRP announcement phase was defined from January 1, 2010 to September 30, 2012 and the post-HRRP 
phase included October 1, 2012 to August 31, 2015. For AECOPD, the HRRP announcement phase included January 1, 2010 to September 30, 2014 and the post-HRRP phase included October 1, 2014 to August 31, 2015. We examined monthly outcome measures for the entire study peroid for each condition and this included 63 observation points. Overall, the equation is represented as follows:

$$
\begin{aligned}
Y_{t}= & \beta_{0}+\beta_{1} * \text { time }_{t}+\beta_{2} * \text { HRRP implementation } \\
& +\beta_{3} * \text { time after HRRP implementation } \\
& +e_{t}
\end{aligned}
$$

$Y_{t}$ is our outcome measure, the rate of readmissions per month in month $t$; time $_{t}$ is a continous variable (163 months), indicating time in month $t$ starting from January 2010 to August 2015. HRRP implementation is a dummy indicator for pre or post HRRP implmentation (before $=0$, after $=1$ ); and the time after HRRP implementation $_{t}$ is a continous variable counting the number of months after the HRRP implementation at time $t$ (before $=0) . \beta_{0}$ estimates the baseline level of the outcome (such as the rate of readmissions per month); $\beta_{1}$ estimates the change in the rate of readmissions per month occuring in each month before HRRP implementation; $\beta_{2}$ estimates the level change in the rate of readmissions per month immediately after HRRP implementation at the end of the preceding segment; and $\beta_{3}$ estimates the change in the trend in the rates of readmissions per month after the HRRP implementation, compared to the trend before the implementation. The error term $e_{t}$ includes random error and autocorrelation. All models were adjusted for seasonality. The sum of $\beta_{1}$ and $\beta_{3}$ was calculated to estimate the post-HRRP implementation slope, and the Wald test was used for hypothesis testing [24].

Index hospitalization and readmission costs for each patient were calculated using standard methods described by the HCUP. These costs were adjusted for inflation to 2015 dollars using the medical component of the consumer price index, and all costs are reported in 2015 dollars [20]. We evaluated differences in readmission costs between early ( $\leq 30$ days) and late ( $>30$ days) readmissions within each targeted condition using a generalized linear model with a log link function and gamma distribution. The cost model was then applied to each targeted condition and furhter stratified by previously defined age groups. Analyses were performed using SAS version 9.4 (SAS Institute, Cary, NC), and all hypothesis testing was two sided with a significance set at $p<0.05$.

\section{Results}

Between January 2010 and September 2015, there were an estimated 2,384,531 hospitalizations nationally for
AMI, 4,491,180 for HF, 5,810,738 for pneumonia, 4,198, 163 for AECOPD, and 93,742,090 for non-targeted conditions. There were an estimated 355,025 (14.89\%) readmissions nationally for AMI, 1,079,016 (24.03\%) readmissions for $\mathrm{HF}, 1,009,849$ (17.38\%) readmissions for pneumonia, 893,376 (21.28\%) readmissions for AECOPD, and 12,564,709 (13.40\%) for non-targeted conditions. The selected demographic characteristics are listed in Table 1. Patients with Medicare were the largest group, and most patients were discharged to home across the targeted and non-targeted conditions. Further breakdowns for patient characteristics of index admissions by age, insurance, and condition are listed in S5 Table.

\section{Readmission trends among targeted conditions prior to and after HRRP implementation Acute myocardial infarction}

Older population ( $\geq 65$ years) The 30 -day readmission rates among aged $\geq 65$ years decreased from $19.79 \%$ in 2010 to $16.56 \%$ in 2015 for AMI (Table 2, Fig. 1a). The slope decreased in the HRRP announcement (slope: $0.0915, p<0.0001$ ) and post-HRRP (slope: $-0.0433, \mathrm{p}<$ 0.0001 ) phases (Table 3). The slope in the HRRP announcement phase was slightly greater compared to the slope in the post-HRRP phase, indicating readmission rates decreased at a slower rate during the post-HRRP phase (difference: 0.0482, $p=0.006$ ). From our DD models, readmission rates declined significantly in the HRRP announcement phase (slope: $-0.0676, p<0.0001$ ), and the rates continued to decrease after HRRP implementation (slope: $-0.0454, p=0.001$ ) (Table 4, Fig. 2a).

Younger population (18-64 years) Similar patterns of readmission rates decline were found in those aged 40-64 years (decreased from 12.19 to $10.7 \%$ ) and 18 39 years (decreased from 10.35 to $8.72 \%$ ) (Table 2, Fig. 1a). A significant decrease in readmission trends were seen in those aged 40-64 years in the HRRP announcement phase (slope: $-0.0385, p<0.0001$ ), right after the HRRP implementation (level change: $0.2882, p=0.04$ ), and post-HRRP (slope: $-0.0159, \mathrm{p}<$ 0.0001) phase (Table 3). In DD models, readmission rates decreased significantly in the HRRP announcement (slope: $-0.0491, p<0.0001$ ) and post-HRRP (slope: $-0.0332, p<0.0001$ ) phases (Table 4, Fig. 2a). Among those aged $18-39$ years, there were no significant changes in baseline readmission trends observed in both ITS (HRRP announcement: $p=0.54$; postHRRP: $p=0.17$ ) and DD models (HRRP announcement: $p=0.37$; post-HRRP: $p=0.19$ ) (Tables 3 and 4). 
Table 1 Demographic and Clinical Characteristics of Index Admissions among Targeted and Non-targeted Condition within the Nationwide Readmissions Database, 2010-2015

\begin{tabular}{|c|c|c|c|c|c|}
\hline Characteristics, No. (\%) & AMI & HF & Pneumonia & COPD & $\begin{array}{l}\text { Non-targeted } \\
\text { Conditions }\end{array}$ \\
\hline Age, year, median (IQR) & $67(57,78)$ & $75(63,84)$ & $73(59,83)$ & $68(59,77)$ & $58(38,74)$ \\
\hline $18-39$ & $56,036(2.3)$ & $104,915(2.3)$ & $379,382(6.5)$ & - & $24,332,212(26.0)$ \\
\hline $40-64$ & $1,009,250(42.3)$ & $1,155,234(25.7)$ & $1,620,305(27.9)$ & $1,652,056(39.4)$ & $32,378,953(34.5)$ \\
\hline$\geq 65$ & $1,319,245(55.4)$ & $3,231,031(72.0)$ & $3,811,051(65.6)$ & $2,546,107(60.6)$ & $37,030,924(39.5)$ \\
\hline \multicolumn{6}{|l|}{ Gender } \\
\hline Male & $1,469,081(61.6)$ & $2,250,158(50.1)$ & $2,813,803(48.4)$ & $1,749,810(41.7)$ & $38,694,908(41.3)$ \\
\hline Female & $915,450(38.4)$ & $2,241,023(49.9)$ & 2,996,935 (51.6) & $2,448,353(58.3)$ & $55,047,181(58.7)$ \\
\hline \multicolumn{6}{|l|}{ Insurance } \\
\hline Medicare & $1,358,056(57.1)$ & $3,416,220(76.1)$ & $4148,871(71.5)$ & $2,961,680(70.7)$ & $43,019,710(46.0)$ \\
\hline Medicaid & $165,552(7.0)$ & $392,824(8.8)$ & $514,536(8.9)$ & $515,798(12.3)$ & $16,644,864(17.8)$ \\
\hline Private & $618,579(26.0)$ & $444,947(10.0)$ & $816,310(14.1)$ & $461,905(11.0)$ & $24,383,243(26.0)$ \\
\hline Self-pay & $141,036(5.9)$ & $125,322(2.8)$ & $175,595(3.0)$ & $129,210(3.1)$ & $93,642(5.8)$ \\
\hline Other & $95,782(4.0)$ & $102,929(2.3)$ & $144,875(2.5)$ & $121,275(2.9)$ & $78,877(4.4)$ \\
\hline \multicolumn{6}{|l|}{ Median household income } \\
\hline$\$ 1-\$ 41,999$ & $722,329(30.8)$ & $1,521,192(34.4)$ & $1,803,527(31.5)$ & $1,542,186(37.3)$ & $28,990,950(31.4)$ \\
\hline$\$ 42,000-\$ 51,999$ & $625,069(26.7)$ & $1,120,857(25.3)$ & $1,486,496(26.0)$ & $1,115,493(27.0)$ & $23,155,075(25.1)$ \\
\hline$\$ 52,000-\$ 67,999$ & $551,102(23.5)$ & $985,561(22.3)$ & $1,323,968(23.1)$ & $874,642(21.2)$ & $21,546,830(23.3)$ \\
\hline$\geq \$ 68,000$ & $446,519(19.0)$ & $798,166(18.0)$ & $1,105,383(19.4)$ & $600,507(14.5)$ & $18,598,519(20.2)$ \\
\hline \multicolumn{6}{|l|}{ Discharge location } \\
\hline Home & $1,703,678(71.5)$ & $2,407,431(53.6)$ & $3,044,937(52.4)$ & $2,743,387(65.3)$ & $66,911,595(71.4)$ \\
\hline Home healthcare & $310,865(13.0)$ & $1,137,616(25.3)$ & $1,039,137(17.9)$ & $815,016(19.4)$ & $11,284,045(12.0)$ \\
\hline SNF/other & $369,988(15.5)$ & $946,133(21.1)$ & $1,726,664(29.7)$ & $639,760(15.3)$ & $15,546,448(16.6)$ \\
\hline No. of comorbidities, median (IQR) & $7(5,9)$ & $8(6,10)$ & $6(4,8)$ & $7(5,9)$ & $4(2,7)$ \\
\hline $\begin{array}{l}\text { Length of stay in days, median } \\
\text { (IQR) }\end{array}$ & $3(2,5)$ & $4(2,6)$ & $4(3,7)$ & $4(2,6)$ & $3(2,5)$ \\
\hline $\begin{array}{l}\text { Total cost for index admission, } \\
\text { median (IQR) }\end{array}$ & $\begin{array}{l}\$ 57,610(\$ 33,195, \\
\$ 95,207)\end{array}$ & $\begin{array}{l}\$ 25,024(\$ 14,514, \\
\$ 45,047)\end{array}$ & $\begin{array}{l}\$ 26,433(\$ 15,068, \\
\$ 48,317)\end{array}$ & $\begin{array}{l}\$ 22,512(\$ 13,382, \\
\$ 39,122)\end{array}$ & $\begin{array}{l}\$ 22,230(\$ 12,415, \\
\$ 41,723)\end{array}$ \\
\hline
\end{tabular}

Abbrev. $A M I$ acute myocardial infarction, $H F$ heart failure, COPD chronic obstructive pulmonary disease, IQR interquartile range

\section{Heart failure}

Older population ( $\geq 65$ years) Thirty-day readmission rates in those aged $\geq 65$ years for HF decreased from 24.83 to $22.53 \%$ from 2010 to 2015 (Table 2, Fig. 1b). Readmission rates declined significantly in the HRRP announcement phase (slope: $-0.0275, p=0.008$ ) (Table 3). Directly after the HRRP implementation, there was a significant decrease in readmission rates (level change: $1.2214, \mathrm{p}<0.0001)$. In the DD models, the readmission trend decline did not reach statistical significance (slope: $-0.0093, \mathrm{p}=0.3$ ); however, the slope was more negative in the post-HRRP phase (slope: $-0.019, p=0.03$ ) [Table 4, Fig. 2b]. In addition, a significant level change was observed (level change: $-0.6902, p=0.003$ ).
Younger population (18-64 years) Baseline readmission trends decline were seen in those aged 40-64 years (decreased from 25.89 to $24.64 \%$ ) (Table 2, Fig. 1b). For those aged 18-39years, the trends decreased from $25.79 \%$ in 2010 to $24.19 \%$ in 2012, going up to $26.30 \%$ in 2013, and then decreased to $24.10 \%$. A significant level change was observed in those aged 40-64 years (level change: $-0.6897, p=0.02$ ), with no significant changes in readmission trends in the HRRP announcement $(p=0.07)$ and post-HRRP phases $(p=0.82)$ (Table 3$)$. In the DD models, readmission rates decreased significantly in the HRRP announcement (slope: $-0.0353, p=0.003)$, and post-HRRP phases $(-0.0216, p=$ $0.05)$ but no significant level change $(p=0.27)$ was observed (Table 4, Fig. 2b). 
Table 2 Number of Index Admissions and 30-day Readmissions Stratified by Targeted and Non-targeted Condition and Age from the Nationwide Readmissions Database, 2010 to 2015 a

\begin{tabular}{|c|c|c|c|c|c|c|c|c|}
\hline \multicolumn{2}{|c|}{ Characteristics } & 2010 & 2011 & 2012 & 2013 & 2014 & 2015 & Overall \\
\hline \multicolumn{9}{|c|}{ Acute Myocardial Infarction } \\
\hline \multirow[t]{3}{*}{ All age groups } & Total index admissions & 401,418 & 400,230 & 416,330 & 422,930 & 418,275 & 325,349 & $2,384,531$ \\
\hline & No. of 30-day readmissions & 65,559 & 63,995 & 61,912 & 60,829 & 57,802 & 44,927 & 355,025 \\
\hline & Readmission rates (\%) & 16.33 & 15.99 & 14.87 & 14.38 & 13.82 & 13.81 & 14.89 \\
\hline \multirow[t]{3}{*}{ 18-39years } & Total index admissions & 9938 & 9939 & 9466 & 9702 & 9392 & 7599 & 56,036 \\
\hline & No. of 30-day readmissions & 1029 & 1017 & 963 & 965 & 874 & 663 & 5510 \\
\hline & Readmission rates (\%) & 10.35 & 10.23 & 10.17 & 9.94 & 9.31 & 8.72 & 9.83 \\
\hline \multirow[t]{3}{*}{ 40-64 years } & Total index admissions & 170,426 & 170,271 & 175,812 & 178,108 & 177,147 & 137,486 & $1,009,250$ \\
\hline & No. of 30-day readmissions & 20,773 & 20,129 & 19,886 & 19,541 & 18,562 & 14,711 & 113,602 \\
\hline & Readmission rates $(\%)$ & 12.19 & 11.82 & 11.31 & 10.97 & 10.48 & 10.70 & 11.26 \\
\hline \multirow[t]{3}{*}{$\geq 65$ years } & Total index admissions & 221,054 & 220,020 & 231,052 & 235,120 & 231,737 & 180,263 & $1,319,246$ \\
\hline & No. of 30-day readmissions & 43,756 & 42,850 & 41,064 & 40,324 & 38,367 & 29,553 & 235,914 \\
\hline & Readmission rates (\%) & 19.79 & 19.48 & 17.77 & 17.15 & 16.56 & 16.39 & 17.88 \\
\hline \multicolumn{9}{|l|}{ Heart Failure } \\
\hline \multirow[t]{3}{*}{ All age groups } & Total index admissions & 785,712 & 767,318 & 751,847 & 767,218 & 790,018 & 629,067 & $4,491,180$ \\
\hline & No. of 30-day readmissions & 197,339 & 191,576 & 182,318 & 179,705 & 182,500 & 145,579 & $1,079,016$ \\
\hline & Readmission rates (\%) & 25.12 & 24.97 & 24.25 & 23.42 & 23.10 & 23.14 & 24.03 \\
\hline \multirow[t]{3}{*}{$18-39$ years } & Total index admissions & 18,099 & 17,510 & 17,433 & 17,841 & 18,857 & 15,176 & 104,916 \\
\hline & No. of 30-day readmissions & 4667 & 4507 & 4510 & 4315 & 4960 & 3658 & 26,617 \\
\hline & Readmission rates $(\%)$ & 25.79 & 25.74 & 25.87 & 24.19 & 26.30 & 24.10 & 25.37 \\
\hline \multirow[t]{3}{*}{ 40-64 years } & Total index admissions & 198,110 & 194,402 & 192,074 & 197,902 & 206,895 & 165,851 & $1,155,234$ \\
\hline & No. of 30-day readmissions & 51,284 & 49,840 & 48,277 & 48,973 & 50,389 & 40,869 & 289,632 \\
\hline & Readmission rates (\%) & 25.89 & 25.64 & 25.13 & 24.75 & 24.35 & 24.64 & 25.07 \\
\hline \multirow[t]{3}{*}{$\geq 65$ years } & Total index admissions & 569,503 & 555,406 & 542,340 & 551,476 & 564,266 & 448,041 & $3,231,032$ \\
\hline & No. of 30-day readmissions & 141,389 & 137,229 & 129,530 & 126,417 & 127,150 & 101,052 & 762,767 \\
\hline & Readmission rates $(\%)$ & 24.83 & 24.71 & 23.88 & 22.92 & 22.53 & 22.55 & 23.61 \\
\hline \multicolumn{9}{|l|}{ Pneumonia } \\
\hline \multirow[t]{3}{*}{ All age groups } & Total index admissions & 971,287 & $1,013,665$ & 991,446 & $1,026,460$ & 998,629 & 809,252 & $5,810,738$ \\
\hline & No. of 30-day readmissions & 176,961 & 181,312 & 175,709 & 174,425 & 167,920 & 133,522 & $1,009,849$ \\
\hline & Readmission rates (\%) & 18.22 & 17.89 & 17.72 & 16.99 & 16.82 & 16.50 & 17.38 \\
\hline \multirow[t]{3}{*}{$18-39$ years } & Total index admissions & 64,872 & 67,535 & 66,124 & 64,078 & 67,980 & 48,793 & 379,382 \\
\hline & No. of 30-day readmissions & 8248 & 8409 & 8489 & 8360 & 8461 & 6272 & 48,239 \\
\hline & Readmission rates (\%) & 12.71 & 12.45 & 12.84 & 13.05 & 12.45 & 12.85 & 12.72 \\
\hline \multirow[t]{3}{*}{ 40-64 years } & Total index admissions & 271,022 & 284,604 & 274,966 & 282,430 & 289,602 & 217,680 & $1,620,304$ \\
\hline & No. of 30-day readmissions & 45,963 & 47,826 & 47,133 & 46,934 & 47,716 & 36,277 & 271,849 \\
\hline & Readmission rates (\%) & 16.96 & 16.80 & 17.14 & 16.62 & 16.48 & 16.67 & 16.78 \\
\hline \multirow[t]{3}{*}{$\geq 65$ years } & Total index admissions & 635,392 & 661,525 & 650,355 & 679,952 & 641,047 & 542,779 & $3,811,050$ \\
\hline & No. of 30-day readmissions & 122,750 & 125,077 & 120,086 & 119,132 & 111,744 & 90,974 & 689,763 \\
\hline & Readmission rates $(\%)$ & 19.32 & 18.91 & 18.46 & 17.52 & 17.43 & 16.76 & 18.10 \\
\hline \multicolumn{9}{|c|}{ Chronic Obstructive Pulmonary Disease } \\
\hline \multirow[t]{3}{*}{ All age groups } & Total index admissions & 729,546 & 757,553 & 730,901 & 733,946 & 697,041 & 549,175 & $4,198,163$ \\
\hline & No. of 30-day readmissions & 159,245 & 166,954 & 156,242 & 150,882 & 145,595 & 114,457 & 893,376 \\
\hline & Readmission rates (\%) & 21.83 & 22.04 & 21.38 & 20.56 & 20.89 & 20.84 & 21.28 \\
\hline
\end{tabular}


Table 2 Number of Index Admissions and 30-day Readmissions Stratified by Targeted and Non-targeted Condition and Age from the Nationwide Readmissions Database, 2010 to 2015ª (Continued)

\begin{tabular}{|c|c|c|c|c|c|c|c|c|}
\hline Characteristics & & 2010 & 2011 & 2012 & 2013 & 2014 & 2015 & Overall \\
\hline \multirow[t]{3}{*}{ 40-64 years } & Total index admissions & 283,859 & 296,422 & 291,524 & 286,029 & 282,095 & 212,126 & $1,652,055$ \\
\hline & No. of 30-day readmissions & 60,293 & 64,070 & 61,161 & 59,086 & 59,441 & 45,440 & 349,491 \\
\hline & Readmission rates $(\%)$ & 21.24 & 21.61 & 20.98 & 20.66 & 21.07 & 21.42 & 21.15 \\
\hline \multirow[t]{3}{*}{$\geq 65$ years } & Total index admissions & 445,687 & 461,131 & 439,377 & 447,918 & 414,946 & 337,049 & $2,546,108$ \\
\hline & No. of 30-day readmissions & 98,952 & 102,884 & 95,081 & 91,797 & 86,155 & 69,017 & 543,886 \\
\hline & Readmission rates (\%) & 22.20 & 22.31 & 21.64 & 20.49 & 20.76 & 20.48 & 21.36 \\
\hline \multicolumn{9}{|c|}{ Non-targeted Conditions } \\
\hline \multirow[t]{3}{*}{ All age groups } & Total index admissions & $16,591,979$ & $16,575,452$ & $16,602,556$ & $16,186,040$ & $16,021,104$ & $11,764,959$ & $93,742,090$ \\
\hline & No. of 30-day readmissions & $2,247,016$ & $2,252,019$ & $2,228,922$ & $2,142,287$ & $2,128,186$ & $1,566,279$ & $12,564,709$ \\
\hline & Readmission rates (\%) & 13.54 & 13.59 & 13.43 & 13.24 & 13.28 & 13.31 & 13.40 \\
\hline \multirow[t]{3}{*}{ 18-39years } & Total index admissions & $4,264,477$ & $4,255,748$ & $4,286,288$ & $4,214,362$ & $4,241,154$ & $3,070,183$ & $24,332,212$ \\
\hline & No. of 30-day readmissions & 416,414 & 415,862 & 425,751 & 412,567 & 412,243 & 300,072 & $2,382,909$ \\
\hline & Readmission rates (\%) & 9.76 & 9.77 & 9.93 & 9.79 & 9.72 & 9.77 & 9.79 \\
\hline \multirow[t]{3}{*}{ 40-64 years } & Total index admissions & $5,796,067$ & $5,782,105$ & $5,770,024$ & $5,561,777$ & 5481,932 & $3,987,048$ & $32,378,953$ \\
\hline & No. of 30-day readmissions & 869,102 & 881,487 & 875,343 & 846,110 & 844,815 & 619,742 & $4,936,599$ \\
\hline & Readmission rates (\%) & 14.99 & 15.25 & 15.17 & 15.21 & 15.41 & 15.54 & 15.25 \\
\hline \multirow[t]{3}{*}{$\geq 65$ years } & Total index admissions & $6,531,435$ & $6,537,600$ & $6,546,243$ & $6,409,902$ & $6,298,017$ & $4,707,728$ & $37,030,925$ \\
\hline & No. of 30-day readmissions & 961,499 & 954,670 & 927,827 & 883,609 & 871,127 & 646,464 & $5,245,196$ \\
\hline & Readmission rates (\%) & 14.72 & 14.60 & 14.17 & 13.79 & 13.83 & 13.73 & 14.16 \\
\hline
\end{tabular}

a Data are included up to August 31, 2015 due to initiation of ICD-10-CM codes in October 1, 2015 within the U.S. coding system

\section{Pneumonia}

Older population ( $\geq 65$ years) Among those aged $\geq 65$ years hospitalized with pneumonia, the overall 30-day readmission rates decreased from $19.32 \%$ in 2010 to $16.76 \%$ in 2015 (Table 2, Fig. 1c). Readmission rates decreased significantly in the HRRP announcement phase (slope: 0.0339, $p<0.0001$ ), immediately after HRRP implementation (level change: $-0.5158, p=0.004$ ), and in the postHRRP phase (slope: $-0.0328, p<0.0001$ ) (Table 3). In the DD models, readmission rates among those aged $\geq 65$ years declined significantly in the HRRP announcement phase (slope: $-0.0128, p=0.05$ ) and declined further in the post-HRRP phase (slope: $-0.033, p<0.0001$ ) (Table 4, Fig. 2c).

Younger population (18-64 years) In younger age groups, the baseline readmission trends in those aged $40-64$ years increased from $16.96 \%$ in 2010 to $17.14 \%$ in 2012 and decreased to $16.67 \%$ in 2015 (Table 2, Fig. 1c). Trends in those aged 18-39 years increased from $12.71 \%$ in 2010 to $13.05 \%$ in 2013 and decreased to $12.85 \%$ in 2015 . Among those aged $40-64$ years, a significantly decrease (level change: -0.6752, $p=$ 0.006) was found after HRRP implementation in the
ITS models but did not reached statistical significance $(p=0.96)$ in the DD models (Tables 3 and 4$)$.

\section{Acute exacerbation of chronic obstructive pulmonary disease}

Older population ( $\geq 65$ years) Readmission rates in those aged $\geq 65$ years for AECOPD decreased from 22.2 to $20.48 \%$ between 2010 and 2015 (Table 2, Fig. 1d). A significant decrease in readmission trends were seen in those aged $\geq 65$ years in the HRRP announcement period (slope: $-0.0360, p=0.005$; Table $3)$. No further significant changes in readmission rates were seen within age groups during the study period. From the DD models, a significant decrease in trends were observed in the HRRP announcement period among those aged $\geq 65$ years (slope: -0.0182 , $p=0.008$ ) (Table 4 and Fig. 2d).

Younger population (18-64 years) Baseline readmission rates in those aged 40-64 years slightly increased from $21.24 \%$ in 2010 to $21.42 \%$ in 2015 (Table 2, Fig. 1d). There were no statistical significance changes observed over the study period from our ITS and DD models in those aged 40-64 years. (Tables 3 and 4). 


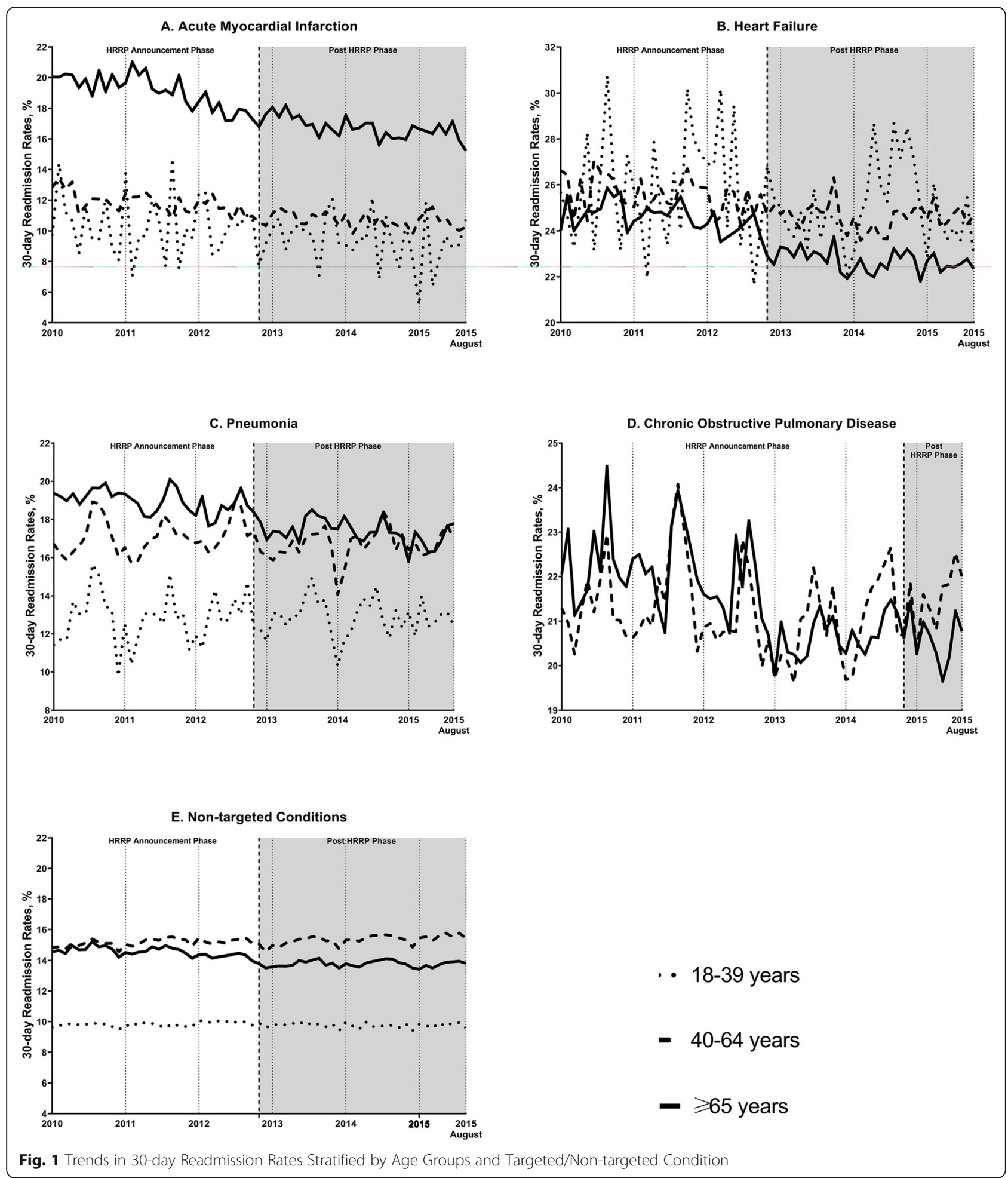

Readmission trends for non-targeted conditions prior and after HRRP implementation

Thirty-day readmission rates for non-targeted conditions remained stable through the study time period (Fig. 1e, Table 2). Readmission trends in those aged $\geq 65$ years decreased in the HRRP announcement phase (slope: $-0.0216, p<0.0001$ ), and a significant decrease was found immediately after the HRRP implementation (level change: $-0.4268, \quad p<0.0001$ ) (Table 3). For those aged 40-64 years, a significant 
Table 3 Interrupted Time Series Analysis Evaluating the Change in 30-day Readmission Rates Prior to and After Implementation of the Hospital Readmission Reduction Program Stratified by Targeted/Non-targeted Condition and Age Group

\begin{tabular}{|c|c|c|c|c|c|c|c|c|}
\hline & $\begin{array}{l}\text { HRRP Announcement Phase } \\
\text { Trend }^{\text {a }} \text { (SE) }\end{array}$ & $P$ & $\begin{array}{l}\text { Level Change }^{b} \\
\text { (SE) }\end{array}$ & $P$ & $\begin{array}{l}\text { Post-HRR Phase } \\
\text { Trend }^{c}\end{array}$ & $P$ & $\begin{array}{l}\text { Difference in } \\
\text { Trends }^{d} \text { (SE) }\end{array}$ & $P$ \\
\hline \multicolumn{9}{|c|}{ Acute Myocardial Infarction } \\
\hline $\begin{array}{l}\text { All age } \\
\text { groups }\end{array}$ & $-0.0697(0.008)$ & $<.0001$ & $-0.2564(0.198)$ & 0.20 & -0.0341 & $<.0001$ & $0.0356(0.01)$ & $\begin{array}{l}< \\
0.001\end{array}$ \\
\hline $\begin{array}{l}18-39 \\
\text { years }\end{array}$ & $-0.0157(0.026)$ & 0.54 & $-0.2452(0.643)$ & 0.70 & -0.0337 & 0.17 & $-0.018(0.035)$ & 0.61 \\
\hline $\begin{array}{l}40-64 \\
\text { years }\end{array}$ & $-0.0385(0.005)$ & $<.0001$ & $-0.2882(0.135)$ & 0.04 & -0.0159 & 0.001 & $0.0226(0.007)$ & 0.001 \\
\hline$\geq 65$ years & $-0.0915(0.01)$ & $<.0001$ & $-0.3971(0.268)$ & 0.14 & -0.0433 & $<.0001$ & $0.0482(0.013)$ & 0.006 \\
\hline \multicolumn{9}{|l|}{ Heart Failure } \\
\hline $\begin{array}{l}\text { All age } \\
\text { groups }\end{array}$ & $-0.0266(0.009)$ & 0.004 & $-1.0354(0.225)$ & $<.0001$ & -0.0126 & 0.14 & $0.014(0.124)$ & 0.26 \\
\hline $\begin{array}{l}18-39 \\
\text { years }\end{array}$ & $0.0157(0.037)$ & 0.67 & $-1.3105(0.961)$ & 0.18 & 0.0196 & 0.58 & $0.0039(0.048)$ & 0.94 \\
\hline $\begin{array}{l}40-64 \\
\text { years }\end{array}$ & $-0.0209(0.011)$ & 0.07 & $-0.6897(0.293)$ & 0.02 & -0.0024 & 0.82 & $0.0185(0.015)$ & 0.022 \\
\hline$\geq 65$ years & $-0.0275(0.010)$ & 0.008 & $-1.2214(0.254)$ & $<.0001$ & -0.017 & 0.06 & $0.0105(0.014)$ & 0.46 \\
\hline \multicolumn{9}{|l|}{ Pneumonia } \\
\hline $\begin{array}{l}\text { All age } \\
\text { groups }\end{array}$ & $-0.0186(0.006)$ & 0.003 & $-0.5478(0.14)$ & 0.0003 & -0.0196 & $\begin{array}{l}< \\
0.001\end{array}$ & $-0.001(0.009)$ & 0.91 \\
\hline $\begin{array}{l}18-39 \\
\text { years }\end{array}$ & $0.0011(0.015)$ & 0.94 & $0.1852(0.383)$ & 0.63 & -0.0077 & 0.59 & $-0.0088(0.019)$ & 0.65 \\
\hline $\begin{array}{l}40-64 \\
\text { years }\end{array}$ & $0.0104(0.009)$ & 0.26 & $-0.6752(0.236)$ & 0.006 & 0.0111 & 0.19 & $0.0008(0.011)$ & 0.95 \\
\hline$\geq 65$ years & $-0.0339(0.007)$ & $<.0001$ & $-0.5158(0.171)$ & 0.004 & -0.0328 & $<.0001$ & $0.0011(0.008)$ & 0.89 \\
\hline \multicolumn{9}{|c|}{ Chronic Obstructive Pulmonary Disease } \\
\hline $\begin{array}{l}\text { All age } \\
\text { groups }\end{array}$ & $-0.0231(0.013)$ & 0.09 & $0.2827(0.606)$ & 0.64 & -0.026 & 0.79 & $-0.0029(0.095)$ & 0.98 \\
\hline $\begin{array}{l}40-64 \\
\text { years }\end{array}$ & $-0.0056(0.015)$ & 0.71 & $0.2427(0.735)$ & 0.74 & 0.0914 & 0.79 & $0.037(0.115)$ & 0.75 \\
\hline$\geq 65$ years & $-0.0360(0.012)$ & 0.005 & $0.2882(0.671)$ & 0.67 & -0.248 & 0.56 & $-0.0212(0.103)$ & 0.84 \\
\hline \multicolumn{9}{|c|}{ Non-targeted Conditions ${ }^{e}$} \\
\hline $\begin{array}{l}\text { All age } \\
\text { groups }\end{array}$ & $-0.0034(0.002)$ & 0.11 & $-0.2706(0.049)$ & $<.0001$ & 0.0026 & 0.21 & $0.006(0.003)$ & 0.06 \\
\hline $\begin{array}{l}18-39 \\
\text { years }\end{array}$ & $0.0071(0.002)$ & 0.002 & $-0.1247(0.051)$ & 0.02 & -0.0031 & 0.15 & $-0.0102(0.003)$ & 0.002 \\
\hline $\begin{array}{l}40-64 \\
\text { years }\end{array}$ & $0.0087(0.006)$ & 0.17 & $-0.1116(0.107)$ & 0.30 & 0.0124 & 0.05 & $0.0037(0.01)$ & 0.71 \\
\hline$\geq 65$ years & $-0.0216(0.003)$ & $<.0001$ & $-0.4268(0.071)$ & $<.0001$ & -0.0016 & 0.59 & $0.02(0.004)$ & $<.0001$ \\
\hline
\end{tabular}

Abbrev. HRRP Hospital Readmissions Reduction Program, SE standard error

${ }^{a}$ The HRRP announcement phase trend represents the change in rates of readmissions per month before HRRP implementation. This was estimated by calculating the $\beta_{1}$ from the equation

${ }^{b}$ The level change represents the level change immediately after HRRP implementation. This was estimated by calculating the $\beta_{2}$ from the equation

c The post-HRRP phase trend represents the post-HRRP implementation slope. This was estimated by calculating the sum of $\beta_{1}$ and $\beta_{3}$ from the equation

discussed in the methods section

${ }^{d}$ The difference in trends represents the trend change after HRRP implementation compared to the trend before implementation. This was estimated by calculating the $\beta_{3}$ from the equation

e The interrupted time series analysis was estimated by using the same HRRP implementation time and age restrictions as the acute myocardial infarction, heart failure and pneumoniia cohorts 
Table 4 DD Estimate of Change in 30-day Readmission Rates Prior to and After Implementation of the Hospital Readmission Reduction Program Between Targeted and Non-targeted Conditions Stratified by Age Groups Using Interrupted Time Series Analysis

\begin{tabular}{|c|c|c|c|c|c|c|c|c|}
\hline & $\begin{array}{l}\text { HRRP Announcement Phase } \\
\text { Trend }^{\text {a }} \text { (SE) }\end{array}$ & $P$ & $\begin{array}{l}\text { Level Change }^{\mathbf{b}} \\
\text { (SE) }\end{array}$ & $P$ & $\begin{array}{l}\text { Post-HRRP Phase } \\
\text { Trend }^{c}\end{array}$ & $P$ & $\begin{array}{l}\text { Difference in } \\
\text { Trends }^{d} \text { (SE) }\end{array}$ & $P$ \\
\hline \multicolumn{9}{|c|}{ Acute Myocardial Infarction } \\
\hline $\begin{array}{l}\text { All age } \\
\text { groups }\end{array}$ & $-0.0638(0.008)$ & $<.0001$ & $0.0042(0.201)$ & 0.98 & -0.0373 & $<.0001$ & $0.0265(0.01)$ & 0.01 \\
\hline $\begin{array}{l}18-39 \\
\text { years }\end{array}$ & $-0.0228(0.025)$ & 0.37 & $-0.0998(0.635)$ & 0.88 & -0.0318 & 0.19 & $-0.009(0.034)$ & 0.8 \\
\hline $\begin{array}{l}40-64 \\
\text { years }\end{array}$ & $-0.0491(0.006)$ & $<.0001$ & $-0.0352(0.158)$ & 0.82 & -0.0332 & $<.0001$ & $0.0159(0.008)$ & 0.05 \\
\hline$\geq 65$ years & $-0.0676(0.014)$ & $<.0001$ & $0.1147(0.338)$ & 0.74 & -0.0454 & 0.001 & $0.0222(0.021)$ & 0.29 \\
\hline \multicolumn{9}{|l|}{ Heart Failure } \\
\hline $\begin{array}{l}\text { All age } \\
\text { groups }\end{array}$ & $-0.0248(0.008)$ & 0.003 & $-0.6847(0.203)$ & 0.001 & -0.0997 & 0.02 & $0.0068(0.011)$ & 0.54 \\
\hline $\begin{array}{l}18-39 \\
\text { years }\end{array}$ & $0.0087(0.037)$ & 0.81 & $-1.2183(0.953)$ & 0.21 & 0.0243 & 0.49 & $0.0156(0.048)$ & 0.74 \\
\hline $\begin{array}{l}40-64 \\
\text { years }\end{array}$ & $-0.0353(0.012)$ & 0.003 & $-0.3339(0.298)$ & 0.27 & -0.0216 & 0.05 & $0.0137(0.015)$ & 0.36 \\
\hline$\geq 65$ years & $-0.0093(0.009)$ & 0.3 & $-0.6902(0.224)$ & 0.003 & -0.019 & 0.03 & $-0.0097(0.012)$ & 0.43 \\
\hline \multicolumn{9}{|l|}{ Pneumonia } \\
\hline $\begin{array}{l}\text { All age } \\
\text { groups }\end{array}$ & $-0.0152(0.006)$ & 0.02 & $-0.2795(0.141)$ & 0.05 & -0.0222 & $\begin{array}{l}< \\
0.001\end{array}$ & $-0.007(0.009)$ & 0.43 \\
\hline $\begin{array}{l}18-39 \\
\text { years }\end{array}$ & $-0.0061(0.014)$ & 0.67 & $0.2977(0.371)$ & 0.43 & -0.004 & 0.77 & $0.0021(0.019)$ & 0.91 \\
\hline $\begin{array}{l}40-64 \\
\text { years }\end{array}$ & $0.001(0.011)$ & 0.92 & $-0.4609(0.273)$ & 0.96 & -0.0044 & 0.66 & $-0.0054(0.014)$ & 0.69 \\
\hline$\geq 65$ years & $-0.0128(0.006)$ & 0.05 & $-0.051(0.164)$ & 0.76 & -0.033 & $<.0001$ & $-0.0202(0.008)$ & 0.002 \\
\hline \multicolumn{9}{|c|}{ Chronic Obstructive Pulmonary Disease } \\
\hline $\begin{array}{l}\text { All age } \\
\text { groups }\end{array}$ & $-0.0168(0.008)$ & 0.04 & $0.2957(0.497)$ & 0.55 & -0.028 & 0.71 & $-0.0112(0.076)$ & 0.88 \\
\hline $\begin{array}{l}40-64 \\
\text { years }\end{array}$ & $-0.0136(0.01)$ & 0.19 & $0.1706(0.613)$ & 0.78 & 0.0247 & 0.8 & $0.0383(0.094)$ & 0.68 \\
\hline$\geq 65$ years & $-0.0182(0.007)$ & 0.008 & $0.4385(0.54)$ & 0.42 & -0.0723 & 0.37 & $-0.0541(0.081)$ & 0.51 \\
\hline
\end{tabular}

Abbrev. DD difference-in-difference, HRRP Hospital Readmissions Reduction Program, SE standard error

${ }^{a}$ The HRRP announcement phase trend represents the change in rates of readmissions per month before HRRP implementation. This was estimated by calculating the $\beta_{1}$ from the equation discussed in the methods section

${ }^{b}$ The level change represents the level change immediately after HRRP implementation. This was estimated by calculating the $\beta_{2}$ from the equation discussed in the methods section

c The post-HRRP phase trend represents the post-HRRP implementation slope. This was estimated by calculating the sum of $\beta_{1}$ and $\beta_{3}$ from the equation discussed in the methods section

${ }^{d}$ The difference in trends represents the trend change after HRRP implementation compared to the trend before implementation. This was estimated by calculating the $\beta_{3}$ from the equation discussed in the methods section

e DD analysis was estimated by using the same HRRP implementation time and age restrictions as the Chronic Obstructive Pulmonary Disease cohort

increase in trend (slope: 0.0124, $p=0.05$ ) was seen in the post-HRRP phase. Among those aged 18-39 years, a significant increase in readmission rates were found in the HRRP announcement phase (slope: $0.0071, p=0.002$ ).

\section{Index hospitalization and readmission costs among the targeted conditions Older population ( $\geq 65$ years)}

Median healthcare expenditures in those aged $\geq 65$ years for readmissions were higher than index hospitalizations for HF, pneumonia, and AECOPD (Fig. 3). For AMI, healthcare expenditures were higher during the index hospitalization. With total expenditures as the outcome, adjusted models showed a significantly higher readmission cost in those readmitted within 30 days (as compared to > 30 days $)$ for AMI $(\$ 15,981.08,95 \%$ CI $[\$ 13$, 712.21-\$18,444.93], $p<0.0001)$, HF $(\$ 16,461.36,95 \%$ CI $[\$ 15,275.93-\$ 17,711.23], p<0.0001)$, pneumonia $(\$ 12$, 622.84, 95\% CI [ $\$ 11,880.55-\$ 13,385.01], p<0.0001)$, and AECOPD (\$10,728.7, 95\% CI [\$10,074.03-\$11,410.21], $p<0.0001)$ (Table 5). 


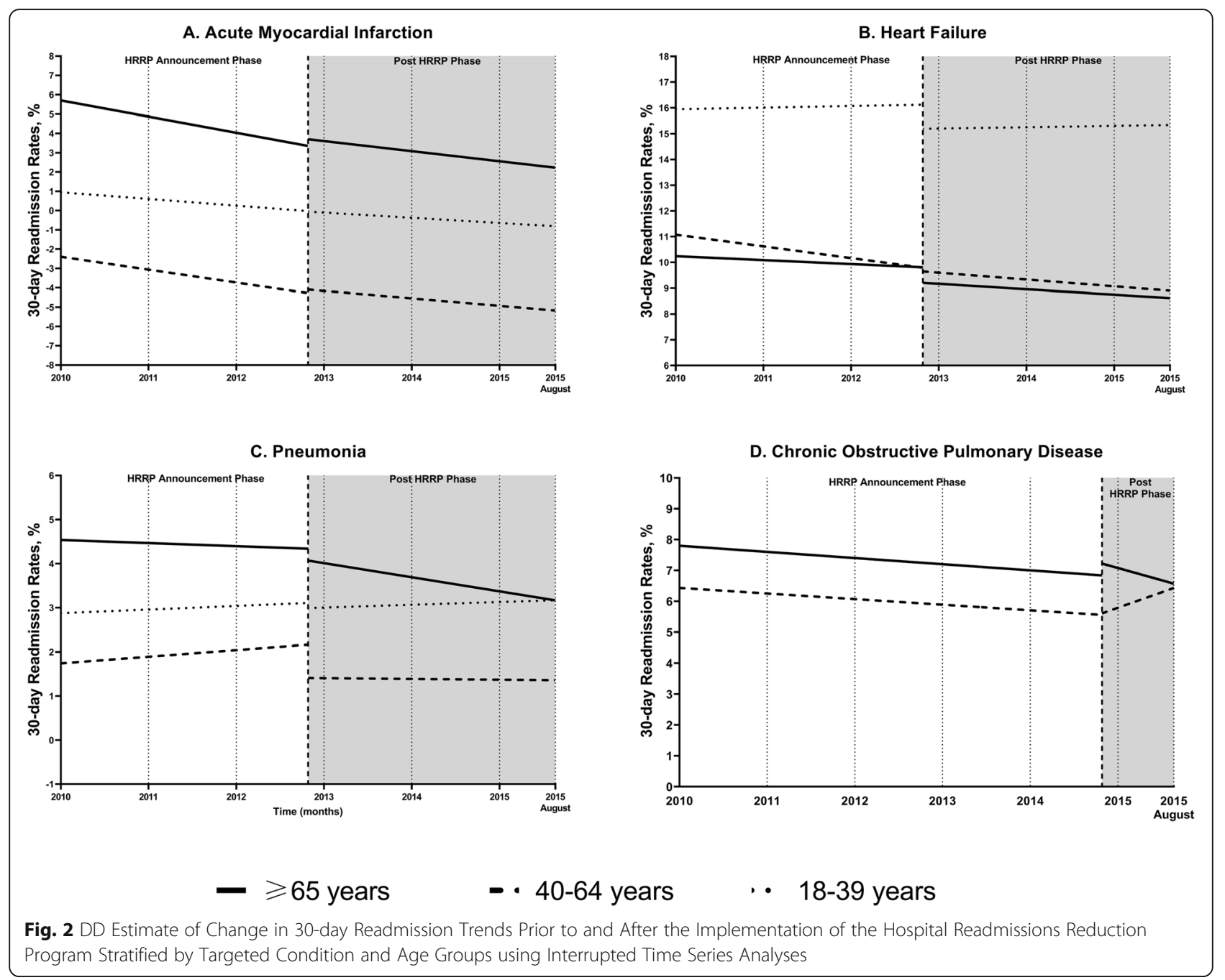

\section{Younger population (18-64 years)}

Among younger age groups median healthcare expenditures for readmissions were higher than index hospitalizations for HF, pneumonia, AECOPD (Fig. 3). The median expenditures of patients aged 40-64 years for readmissions were higher than other age groups across all targeted conditions. For total expenditures, the cost differences among those aged 18-39 years for HF ( $\$ 20$, 035.9, 95\%CI [\$15,401.37-\$25,548.43], $p<0.0001)$ and pneumonia $(\$ 12,867.63,95 \% \mathrm{CI}[\$ 10,991.28-\$ 14,937.81]$, $p<0.0001$ ) were larger than other age groups (Table 5).

\section{Discussion}

This study used a nationwide representative dataset from 2010 to 2015 to provide estimates of hospital readmissions for the major four HRRP conditions prior to and after policy implementation. Overall, 30-day readmission rates in those aged $\geq 65$ years for AMI and pneumonia decreased before and after policy implementation based on ITS and DD models. Similar trend patterns were found in those aged 40-64 years for AMI in both models, and for HF in the DD models only. After adjustment for covariates, healthcare expenditures in those aged $\geq 65$ years and $40-64$ years were significantly higher for those readmitted within 30 days for all targeted indications. The cost differences of healthcare expenditures were largest in those aged 18-39years for HF and pneumonia.

Previous studies have examined the patient characteristics of readmission patterns among younger adults in the original 3 targeted conditions (AMI, HF, and pneumonia) but limited to single year or selected state $[6,7]$. Studies also reported readmission burden in younger adults for those 3 conditions, approximately 1 in 12 patients with AMI, 1 in 5 patients with HF, and 1 in 7 patients with pneumonia aged $<65$ years were readmitted within 30-days of discharge [7, 25]. Our results extend previous articles and also support Angraal et al. work by confirming the decreased readmission trends before and after the HRRP implementation among younger and 


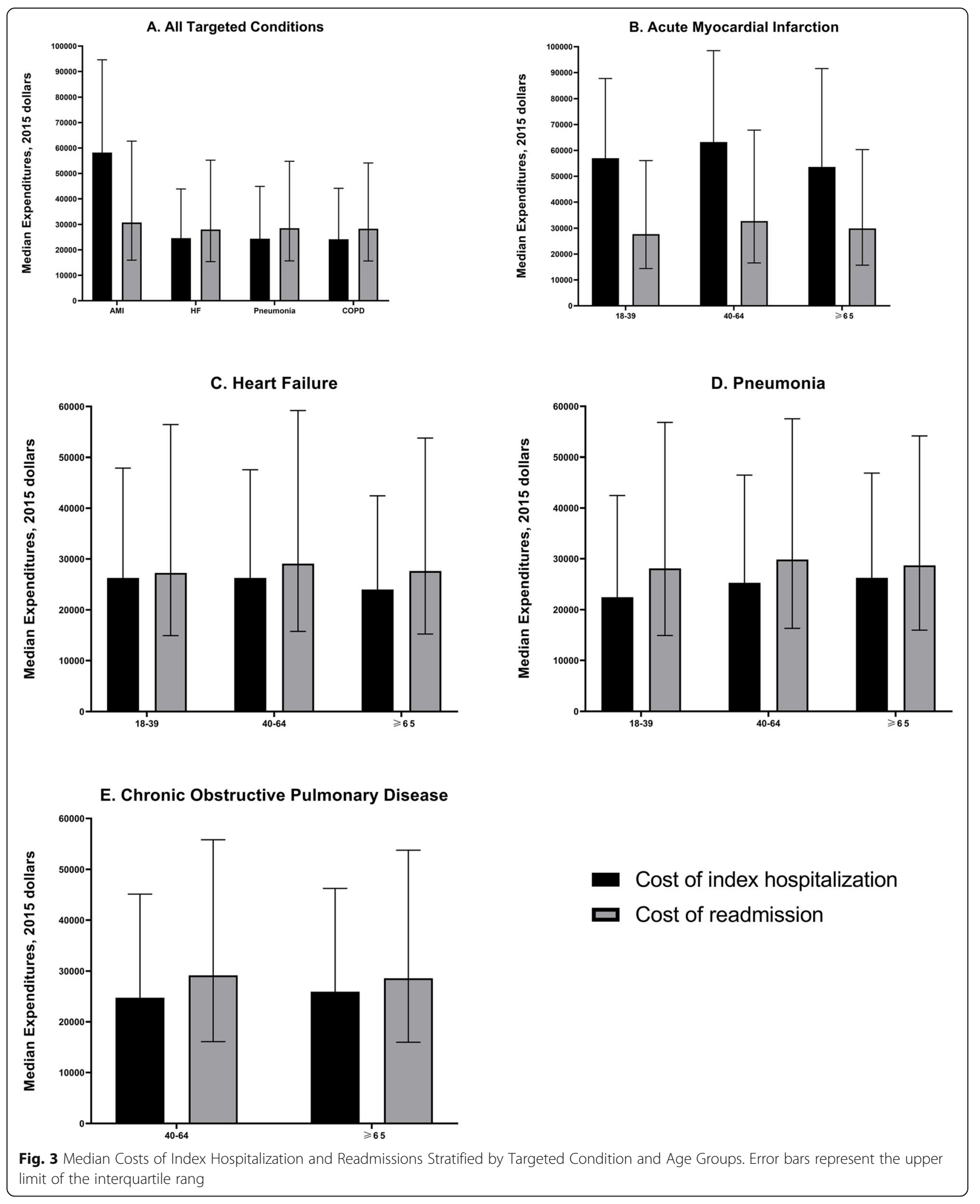


Table 5 Cost Difference Between Early ( $\leq 30$ days) and Late (> 30 days) Readmission Events Stratified by Condition and Age

\begin{tabular}{|c|c|c|c|c|}
\hline & \multicolumn{2}{|l|}{ Unadjusted model $^{\text {a }}$} & \multicolumn{2}{|l|}{ Adjusted model ${ }^{a, b}$} \\
\hline & Cost difference $(95 \% \mathrm{Cl})$ & $P$ & Cost difference $(95 \% \mathrm{Cl})$ & $P$ \\
\hline \multicolumn{5}{|c|}{ Acute Myocardial Infarction } \\
\hline All age groups & $\$ 4327.92(\$ 3902.73-\$ 4762.70)$ & $<0.0001$ & $\$ 4974.12(\$ 4388.94-\$ 5572.89)$ & $<0.0001$ \\
\hline 18-39years & $\$-1043.86(\$-3611.68-\$ 1879.17)$ & 0.47 & $\$-1211.50(\$-2944.96-\$ 1845.86)$ & 0.37 \\
\hline 40-64 years & $\$ 3364(\$ 2604.52-\$ 4148.07)$ & $<0.0001$ & $\$ 1403.63(\$ 982.66-\$ 1874.51)$ & $<0.0001$ \\
\hline$\geq 65$ years & $\$ 5044(\$ 4522.25-\$ 5571.34)$ & $<0.0001$ & $\$ 15,981.08(\$ 13,712.21-\$ 18,444.93)$ & $<0.0001$ \\
\hline \multicolumn{5}{|l|}{ Heart Failure } \\
\hline All age groups & $\$ 6448.47(\$ 6260.52-\$ 6753.10)$ & $<0.0001$ & $\$ 8214.33(\$ 7805.49-\$ 8631.4)$ & $<0.0001$ \\
\hline 18-39years & $\$ 13,362.79(\$ 11,110.16-\$ 15,762.72)$ & $<0.0001$ & $\$ 20,035.9(\$ 15,401.37-\$ 25,548.43)$ & $<0.0001$ \\
\hline 40-64 years & $\$ 7512.03(\$ 6969-\$ 8066.45)$ & $<0.0001$ & $\$ 4623.18(\$ 4157.55-\$ 5120.16)$ & $<0.0001$ \\
\hline$\geq 65$ years & $\$ 5752.42(\$ 5481.69-\$ 6029.36)$ & $<0.0001$ & $\$ 16,461.36(\$ 15,275.93-\$ 17,711.23)$ & $<0.0001$ \\
\hline \multicolumn{5}{|l|}{ Pneumonia } \\
\hline All age groups & $\$ 8856.43(\$ 8610.4-\$ 9109.65)$ & $<0.0001$ & $\$ 8027.16$ (\$7726.24 - \$8338.59) & $<0.0001$ \\
\hline 18-39years & $\$ 12,745.66(\$ 11,419.96-\$ 14,122.99)$ & $<0.0001$ & $\$ 12,867.63(\$ 10,991.28-\$ 14,937.81)$ & $<0.0001$ \\
\hline 40-64 years & $\$ 9890.97(\$ 9373.67-\$ 10,419.55)$ & $<0.0001$ & $\$ 6020.75(\$ 5523.78-\$ 6553)$ & $<0.0001$ \\
\hline$\geq 65$ years & $\$ 8228.76(\$ 7944.76-\$ 8518.59)$ & $<0.0001$ & $\$ 12,622.84(\$ 11,880.55-\$ 13,385.01)$ & $<0.0001$ \\
\hline \multicolumn{5}{|c|}{ Chronic Obstructive Pulmonary Disease } \\
\hline All age groups & $\$ 8390.38(\$ 8148.52-\$ 8633.39)$ & $<0.0001$ & $\$ 6642.03(\$ 6391.26-\$ 6901.31)$ & $<0.0001$ \\
\hline 40-64 years & $\$ 7754.06(\$ 7283.05-\$ 8229.44)$ & $<0.0001$ & $\$ 5107.02(\$ 4664.35-\$ 5562.89)$ & $<0.0001$ \\
\hline$\geq 65$ years & $\$ 7751.64(\$ 7464.94-\$ 8039.25)$ & $<0.0001$ & $\$ 10,728.7(\$ 10,074.03-\$ 11,410.21)$ & $<0.0001$ \\
\hline
\end{tabular}

${ }^{a}$ Standardized to 2015 U.S. dollars

${ }^{\mathrm{b}}$ Adjusted for age, gender, median household income, and Elixhauser comorbidity index scores

older populations [26]. We further stratified the younger populations into 2 age groups (18-39 years and 40-64 years) for three targeted conditions and the rates of readmission decline were observed in those aged 40-64 years for AMI in ITS and DD models, and for HF in DD models (Tables 3 and 4). Myers et al. examined the association between the AECOPD and HRRP and showed a modest decrease in all-cause readmission prior to AECOPD being included as a targeted diagnosis, which is consistent with our findings [27]. Furthermore, our results include larger sample size than previous studies and perform the longitudinal analysis in younger adults (40-64 years) and older adults for AECOPD separately and find similar patterns of decreased readmission rates in both populations in the HRRP announcement phase $[27,28]$. We believe it is possibly that reducing readmissions was already a nationwide concern far before the PPACA and hospitals already responded to reduce readmission rates before policy implementation.

There is currently limited literature that evaluates the cost burden of hospitalizations and readmissions for AMI, HF, pneumonia, and AECOPD. A study by Jain et al. used the NRD to evaluate pneumonia hospitalizations and found mean cost per readmission was highest for the 40-64 age group $(\$ 15,976)$ [29]. A separate study evaluated COPD using the Florida State Inpatient
Database and found mean total charges for 30-day COPD related readmissions increased from 2009 (\$36, $714)$ to $2014(\$ 40,611 ; p=0.011)$ [30]. Finally Khera et al. evaluated AMI using the NRD and found cumulative costs of 30 day readmissions was $\$ 1.1$ billion, of which $\$ 365$ million was among those $<65$ years of age [25]. Our findings extend this work to the four targeted therapeutic conditions including older and younger age groups. We show that the median costs of index hospitalizations among younger age groups are slightly higher or close to the costs in the older populations across the four targeted conditions. This supports that the cost burden to the healthcare system among younger populations is substantial and should be addressed in future research. Based on our adjusted cost difference models, we found that healthcare expenses differences are seen within therapeutic condition as well as specific age groups. These factors should be accounted for in future policy with necessary changes made periodically based on the outcomes in each condition and age group in order to decrease healthcare costs.

The HRRP financially incentivizes hospitals to reduce excess readmissions among fee-for-service Medicare patients aged $\geq 65$ years $[2,3]$. Previous studies have shown a decrease in 30-day hospital readmissions in Medicare patients for the initial targeted conditions (e.g., AMI, 
HF, and pneumonia) under the HRRP. Our baseline trends of 30-day all-cause readmission rates are consistent with the existing literature for these initial targeted conditions among older populations. Whether the decrease in readmission rates is due to the HRRP policy or alternative explanations is controversial. McWilliams et al. suggested a concurrent decline in admission rates during this time period, which may explain much of the reduction in readmission rates for targeted and nontargeted conditions by the HRRP [31]. Another proposed explanation is that the majority of the decrease in readmission was generated by increased patient risk scores, rather than by actual lower readmission rates [32, 33]. The Centers for Medicare and Medicaid Services changed the electronic standards that hospitals used to submit Medicare claims starting in 2011. This increased the number of secondary diagnoses reporting from 1025 diagnosis codes, which allowed for the coding of additional comorbid conditions. After accounting for this change, this would reduce the decline in risk-adjusted readmission rates for targeted conditions by $48 \%$. In addition, we found that readmission rates declined in select age groups outside of the targeted $\geq 65$ years Medicare group. One potential reason for declining readmission rates for those $<65$ years may be due to a spillover effect of the policy beyond the Medicare population, which has been previously described in nontargeted Medicare conditions, Medicaid and private insurance populations $[8,26,34,35]$. Our findings support the decline in readmission rates from 2010 to 2015 for targeted conditions among older adults and in some cases younger adults. The extent to which these declines are due to the HRRP policy or attributed to alternative explanations is still unclear.

The HRRP policy expanded in October 2014 to include AECOPD; however, at the time, there was a lack of published evidence that pointed to effective hospitalbased programs to reduce COPD-related readmissions [36]. Given the financial penalties instituted by the CMS for 30-day readmissions, it is probable that hospitals across the U.S. were implementing quality improvement programs in these targeted areas. Interestingly, several studies have shown limited success at reducing readmissions after a hospitalization for COPD. Jennings et al. performed a single-center, randomized clinical trial evaluating a pre-discharge bundle for COPD exacerbations using a low resource approach and found no differences in readmission rates [37]. A significant limitation was that a tool designed to identify COPD readmission risk factors was ineffective, as it did not include specific interventions or patient follow-up; interventions for identified risk factors were left to the judgement of the primary team. Bhatt et al. performed a single-center, pre-post intervention study evaluating a comprehensive
COPD multidisciplinary intervention focusing on inpatient, transitional, and outpatient care [38]. The intervention did not reduce 30-day readmission rates or overall costs. Finally, Aboumatar et al. performed a single-center, randomized clinical trial, initiating a threemonth program that combined transition support with long-term disease self-management support for patients admitted for AECOPD [39]. The program resulted in significantly more COPD-related hospitalizations and emergency department visits without improvements in quality of life. A sustained decrease in COPD-related readmissions may be interrupted without specific evidence-based interventions showing success in reducing these readmissions. There is a need for continued surveillance as additional data become available and the impact of the HRRP on AECOPD readmissions can be further elucidated.

The penalties associated with the HRRP are quite high, as hospitals could lose up to $3 \%$ of their total fee-forservice Medicare payments due to poor performance on the readmission metric [2]. Readmission-specific incentives and initiatives may have contributed to the decrease in readmissions rates for the targeted conditions. As programs were announced to curb readmission rates, the added interventions may have influenced patient care within Medicare or beyond Medicare patients through increased awareness of changes in the discharge process. For example, Ody et.al reported that changing the CMS Medicare electronic transaction standards allows documenting more diagnosis codes per claim between 2010 and 2012, which may have led to changes on nationwide readmissions and current coding practice in hospitals [32]. Even with the pronounced decrease in readmissions since policy implementation, $83 \%$ of hospitals were penalized under this program for fiscal year 2020 [3]. In addition, evidence is emerging that postdischarge healthcare utilization is increasing (e.g., observation units and emergency department visits) with the subsequent decrease in inpatient readmissions [40]. These increases could be attributable to hospitals treating patients who return within 30 days of discharge in these units, as these are not evaluated under the HRRP policy. Further research is necessary evaluating total healthcare utilization following hospital discharge including emergency department visits, observation units, and hospital readmissions in order to better understand the overall impact of the HRRP on the U.S. healthcare system.

The findings of our study should be interpreted in the context of important limitations. First, we relied on CMS algorithms using ICD-9-CM codes to classify hospitalizations for our targeted cohorts. The selections for the ICD-9-CM codes based algorithms may have led to an underestimation of the number of hospitalizations for 
the targeted conditions [41]. Since this methodology is used by the CMS to identify hospital admissions, we felt it was prudent to apply it to the entire study to provide national readmission estimates across age groups. Due to a limitation in the NRD, we excluded patients who were residents of different states. Persons are identified and tracked in the NRD by state-specific linkage numbers; therefore, a person readmitted between two different states cannot be tracked between states. In addition, we did not include data after October 1, 2015, since the United States newly implemented the ICD-10-CM coding system. It is uncertain whether the transition from ICD-9-CM to ICD-10-CM affects the coding of our targeted conditions. In addition, the first year of available NRD data is 2010, therefore we could not account for readmission rate changes in the pre-2010 period. Previous research has shown a readmission trend change in 2010 as a consequence of the passage of the PPACA [3]. Due to the nature of the NRD, the hospitalizations can only be linked within a year. Thus, the follow up window of late readmission for index hospitalizations occurred later in the year may not be consistent which may lead to under/overestimate the results of the cost difference between early and late readmissions. In 2016, the twenty-first Century Cures Act introduced a new payment method for the HRRP program starting fiscal year 2019 (October 1, 2018 to September 30, 2019) [42-44]. Our study did not reflect this new payment method, however future longitudinal research is needed to evaluate the performance of this new policy for all targeted conditions and age groups.

\section{Conclusions}

Readmissions in those aged $\geq 65$ years for targeted conditions including AMI and pneumonia decreased in the U.S. prior to and after implementation of the HRRP policy. A decrease in hospital readmissions was seen in younger age groups (aged 40-64 years) for AMI and HF not originally targeted by the program. Healthcare expenditures in those aged $\geq 65$ years and $40-64$ years for early readmissions were significantly higher for all targeted conditions. Further research is needed into whether a similar downward trend is occurring beyond hospital admissions including additional markers of healthcare utilization such as observation unit and emergency department visits.

\footnotetext{
Abbreviations

HRRP: Hospital readmissions reduction program; PPACA: Patient protection and affordable care act; AMI: Acute myocardial infarction; HF: Heart failure; AECOPD: Acute exacerbations of chronic obstructive pulmonary disease; FY: Fiscal year; NRD: Nationwide Readmission Database; AHRQ: Agency for Healthcare Research and Quality; HCUP: Healthcare Cost and Utilization Project; ICD-9-CM: International Classification of Diseases, Ninth Revision, Clinical Modification; CMS: Centers for Medicare and Medicaid Services; DD: Difference-in-difference
}

\section{Supplementary Information}

The online version contains supplementary material available at https://doi. org/10.1186/s12913-021-06399-z.

Additional file 1: Table S1. ICD-9-CM Codes Used to Define Acute Myocardial Infarction Cohort. Table S2. ICD-9-CM Codes Used to Define Heart Failure Cohort. Table S3. ICD-9-CM Codes Used to Define Pneumonia Cohort. Table S4. ICD-9-CM Codes Used to Define Chronic Obstructive Pulmonary Disease. Cohort. Table S5. Baseline Patient

Characteristics of Index Admissions by Age Group, Insurance Type, and Targeted Condition. Table S1-S4 include the ICD-9-CM codes for each cohort base on those published by the Centers for Medicare and Medicaid Services for the HRRP for assessment of all cause readmissions. Table S5 includes further breakdowns for patient characteristics of index admissions by age, insurance, and condition.

\section{Acknowledgements}

Not applicable.

\section{Authors' contributions}

$\mathrm{DMJ}$ and $\mathrm{CHL}$ conceptualized and designed the study. $\mathrm{CHL}, \mathrm{CMC}, \mathrm{RT}, \mathrm{MH}$, WG, DMJ collected and analyzed the data. CHL, CMC, RT, WH, EB, CJD, and DMJ interpreted the analysis. CHL, CMC, and DMJ drafted the manuscript. $\mathrm{CHL}$ and DMJ critically revised the manuscript. All authors gave final approval of the manuscript.

\section{Funding}

None.

Availability of data and materials

All relevant data is available from the Nationwide Readmissions Database through the AHRQ Healthcare Cost and Utilization Project. The data are publicly available and could be purchased online through the AHRQ.

\section{Declarations}

Ethics approval and consent to participate Not applicable.

\section{Consent for publication}

Not applicable.

\section{Competing interests}

The authors declare that they have no competing interests.

Received: 28 April 2020 Accepted: 15 April 2021

Published online: 26 April 2021

\section{References}

1. Jencks SF, Williams MV, Coleman EA. Rehospitalizations among patients in the Medicare fee-for-service program. N Engl J Med 2009;360(14):14181428. Epub 2009/04/03. doi: https://doi.org/10.1056/NEJMsa0803563. PubMed PMID: 19339721.

2. Zuckerman RB, Sheingold SH, Orav EJ, Ruhter J, Epstein AM. Readmissions, observation, and the hospital readmissions reduction program. N Engl J Med 2016;374(16):1543-1551. Epub 2016/02/26. doi: https://doi.org/10.1056/ NEJMsa1513024. PubMed PMID: 26910198.

3. Desai NR, Ross JS, Kwon JY, Herrin J, Dharmarajan K, Bernheim SM, et al. Association Between Hospital Penalty Status Under the Hospital Readmission Reduction Program and Readmission Rates for Target and Nontarget Conditions. JAMA. 2016; 316(24): 2647-2656. Epub 2016/12/28. doi: https://doi.org/10.1001/jama.2016.18533. PubMed PMID: 28027367; PubMed Central PMCID: PMCPMC5599851.

4. Strom JB, Kramer DB, Wang Y, Shen C, Wasfy JH, Landon BE, et al. Shortterm rehospitalization across the spectrum of age and insurance types in the United States. PLoS One. 2017; 12(7): e0180767. Epub 2017/07/13. doi: https://doi.org/10.1371/journal.pone.0180767. PubMed PMID: 28700736; PubMed Central PMCID: PMCPMC5507267.

5. Fingar K, Washington R. Trends in Hospital Readmissions for Four HighVolume Conditions, 2009-2013: Statistical Brief \#196. Healthcare Cost and 
Utilization Project (HCUP) Statistical Briefs. Rockville: Agency for Healthcare Research and Quality (US); 2006.

6. Ranasinghe I, Wang Y, Dharmarajan K, Hsieh AF, Bernheim SM, Krumholz HM. Readmissions after hospitalization for heart failure, acute myocardial infarction, or pneumonia among young and middle-aged adults: a retrospective observational cohort study. PLoS Med. 2014; 11(9): e1001737. Epub 2014/10/01. doi: https://doi.org/10.1371/journal.pmed.1001737. PubMed PMID: 25268126; PubMed Central PMCID: PMCPMC4181962.

7. Sukul D, Sinha SS, Ryan AM, Sjoding MW, Hummel SL, Nallamothu BK. Patterns of Readmissions for Three Common Conditions Among Younger US Adults. Am J Med. 2017;130(10):1220. e1-e16. https://doi.org/10.1016/j.a mjmed.2017.05.025.

8. Ferro EG, Secemsky EA, Wadhera RK, Choi E, Strom JB, Wasfy JH, et al. Patient readmission rates for all insurance types after implementation of the hospital readmissions reduction program. Health Affairs (Project Hope). 2019;38(4):585-93. Epub 2019/04/02. 30933582. https://doi.org/10.1377/hltha ff.2018.05412.

9. Leppin AL, Gionfriddo MR, Kessler M, Brito JP, Mair FS, Gallacher K, et al. Preventing 30-day hospital readmissions: a systematic review and metaanalysis of randomized trials. JAMA Intern Med. 2014; 174(7): 1095-1107. Epub 2014/05/14. doi: https://doi.org/10.1001/jamainternmed.2014.1608. PubMed PMID: 24820131; PubMed Central PMCID: PMCPMC4249925.

10. Slazak E, Cardinal C, Will S, Clark CM, Daly CJ, Jacobs DM. Pharmacist-led transitions-of-care services in primary care settings: opportunities, experiences, and challenges. J Am Pharm Assoc (2003). 2019. Epub 2019/12/ 24. doi: https://doi.org/10.1016/j.japh.2019.11.016. PubMed PMID: 31866384, $3,443,449$.

11. Slazak E, Shaver A, Clark CM, Cardinal C, Panthapattu M, Prescott WA, Jr., et al. Implementation of a Pharmacist-Led Transitions of Care Program within a Primary Care Practice: A Two-Phase Pilot Study. Pharmacy (Basel). 2020; 8(1). Epub 2020/01/18. doi: https://doi.org/10.3390/pharmacy8010004. PubMed PMID: 31947920.

12. Ensing HT, Stuijt CC, van den Bemt BJ, van Dooren AA, Karapinar-Carkit F, Koster ES, et al. Identifying the Optimal Role for Pharmacists in Care Transitions: A Systematic Review. J Manag Care Spec Pharm. 2015; 21(8): 614-636. Epub 2015/08/04. doi: https://doi.org/10.18553/jmcp.2015.21.8.614. PubMed PMID: 26233535.

13. Agency for Healthcare Research and Quality. Healthcare cost and utilization project: introduction to the HCUP Nationwide Readmissions Database (NRD) 2010-2015. Rockville: Agency of Healthcare Research and Quality 2017 [cited 2019 August 7]. Available from: https://www.hcup-us.ahrq.gov/db/na tion/nrd/Introduction_NRD_2010-2015.pdf.

14. Mehta AB, Douglas IS, Walkey AJ. Hospital Noninvasive Ventilation Case Volume and Outcomes of Acute Exacerbations of Chronic Obstructive Pulmonary Disease. Ann Am Thoracic Soc. 2016; 13(10): 1752-1759. Epub 2016/07/13. doi: https://doi.org/10.1513/AnnalsATS.201603-2090C. PubMed PMID: 27404021; PubMed Central PMCID: PMCPMC5122492.

15. Yale University/Yale-New Haven Hospital-Center for Outcomes Research \& Evaluation. Hospital 30-Day Acute Myocardial Infarction Readmission Measure: Methodology [cited 2019 May 7]. Available from: https://www.qua litynet.org/dcs/ContentServer?cid=\%201219069855841\&pagename= QnetPublic\%2FPage\%2FQnetTier3\&c=Page.

16. Yale University/Yale-New Haven Hospital-Center for Outcomes Research \& Evaluation. Hospital 30-Day Heart Failure Readmission Measure: Methodology [cited 2019 May 7]. Available from: https://www.qualitynet. org/dcs/ContentServer?cid=\%201219069855841\&pagename=QnetPublic\%2 FPage\%2FQnetTier3\&c=Page.

17. Yale University/Yale-New Haven Hospital-Center for Outcomes Research \& Evaluation. Hospital 30-Day Pneumonia Readmission Measure: Methodology [cited 2019 May 7]. Available from: https://www.qualitynet.org/dcs/ ContentServer?cid=\%201219069855841\&pagename=QnetPublic\%2FPage\%2 FQnetTier $3 \& c=$ Page.

18. Yale University/Yale-New Haven Hospital-Center for Outcomes Research \& Evaluation. Hospital 30-Day 30-day Readmission Following Admission for an Acute Exacerbation of Chronic Obstructive Pulmonary Disease Measure Methodology Report [cited 2019 May 7]. Available from: https://www.qua litynet.org/dcs/ContentServer?cid=\%201219069855841\&pagename= QnetPublic\%2FPage\%2FQnetTier3\&c=Page.

19. Elixhauser A, Steiner C, Harris DR, Coffey RM. Comorbidity measures for use with administrative data. Med Care. 1998;36(1):8-27.
20. U.S. Bureau of labor and statistics. CPI for All Urban Consumers [cited 2019 May 7]. Available from: https://data.bls.gov/timeseries/CUURO000SA M?output_view=data.

21. Penfold $R B$, Zhang $F$. Use of interrupted time series analysis in evaluating health care quality improvements. Acad Pediatr 2013;13(6 Suppl):S38-S44. Epub 2013/12/07. doi: https://doi.org/10.1016/j.acap.2013.08.002. PubMed PMID: 24268083

22. Mascha EJ, Sessler DI. Segmented regression and difference-in-difference methods: assessing the impact of systemic changes in health care. Anesth Analg 2019;129(2):618-633. Epub 2019/04/23. doi: https://doi.org/10.1213/a ne.0000000000004153. PubMed PMID: 31008746.

23. Du DT, Zhou EH, Goldsmith J, Nardinelli C, Hammad TA. Atomoxetine use during a period of FDA actions. Med Care 2012;50(11):987-992. Epub 2012/ 10/11. doi: https://doi.org/10.1097/MLR.0b013e31826c86f1. PubMed PMID: 23047788.

24. Wagner AK, Soumerai SB, Zhang F, Ross-Degnan D. Segmented regression analysis of interrupted time series studies in medication use research. J Clin Pharm Ther 2002;27(4):299-309. Epub 2002/08/14. PubMed PMID: 12174032, DOl: https://doi.org/10.1046/j.1365-2710.2002.00430.x

25. Khera R, Jain S, Pandey A, Agusala V, Kumbhani DJ, Das SR, et al. Comparison of readmission rates after acute myocardial infarction in 3 patient age groups (18 to 44, 45 to 64, and $\geq 65$ years) in the United States. Am J Cardiol. 2017; 120(10):1761-7. https://doi.org/10.1016/.jamjcard.2017.07.081.

26. Angraal S, Khera R, Zhou S, Wang Y, Lin Z, Dharmarajan K, et al. Trends in 30-Day Readmission Rates for Medicare and Non-Medicare Patients in the Era of the Affordable Care Act. Am J Med. 2018; 131(11): 1324-1331.e14. Epub 2018/07/18. doi: https://doi.org/10.1016/j.amjmed.2018.06.013. PubMed PMID: 30016636; PubMed Central PMCID: PMCPMC6380174.

27. Myers LC, Faridi MK, Hasegawa K, Hanania NA, Camargo CA, Jr. The Hospital Readmissions Reduction Program and Readmissions for Chronic Obstructive Pulmonary Disease, 2006-2015. Ann Am Thorac Soc 2019. Epub 2019/12/21. doi: https://doi.org/10.1513/AnnalsATS.201909-672OC. PubMed PMID: 31 $860333,17,4,2456$.

28. Jacobs DM, Noyes K, Zhao J, Gibson W, Murphy TF, Sethi S, et al. Early Hospital Readmissions after an Acute Exacerbation of Chronic Obstructive Pulmonary Disease in the Nationwide Readmissions Database. Ann Am Thoracic Soc. 2018; 15(7): 837-845. Epub 2018/04/04. doi: https://doi.org/1 0.1513/AnnalsATS.201712-9130C. PubMed PMID: 29611719; PubMed Central PMCID: PMCPMC6207114

29. Jain $S$, Khera R, Mortensen EM, Weissler JC. Readmissions of adults within three age groups following hospitalization for pneumonia: Analysis from the Nationwide Readmissions Database. PloS One. 2018; 13(9): e0203375. Epub 2018/09/14. doi: https://doi.org/10.1371/journal.pone.0203375. PubMed PMID: 30212485; PubMed Central PMCID: PMCPMC6136736.

30. Jiang X, Xiao H, Segal R, Mobley WC, Park H. Trends in Readmission Rates, Hospital Charges, and Mortality for Patients With Chronic Obstructive Pulmonary Disease (COPD) in Florida From 2009 to 2014. Clin Ther. 2018; 40(4): 613-626.e1. Epub 2018/04/04. doi: https://doi.org/10.1016/j.clinthera.2 018.03.006. PubMed PMID: 29609879.

31. McWilliams JM, Barnett ML, Roberts ET, Hamed P, Mehrotra A. Did hospital readmissions fall because per capita admission rates fell? Health Affairs (Project Hope). 2019;38(11):1840-4. https://doi.org/10.1377/hlthaff.2019. 00411.

32. Ody C, Msall L, Dafny LS, Grabowski DC, Cutler DM. Decreases in readmissions credited to Medicare's program to reduce hospital readmissions have been overstated. Health Affairs (Project Hope). 2019;38(1): 36-43. Epub 2019/01/08. doi: https://doi.org/10.1377/hlthaff.2018.05178. PubMed PMID: 30615522.

33. Ibrahim AM, Dimick JB, Sinha SS, Hollingsworth JM, Nuliyalu U, Ryan AM. Association of Coded Severity With Readmission Reduction After the Hospital Readmissions Reduction Program. JAMA Intern Med. 2018; 178(2): 290-292. Epub 2017/11/14. doi: https://doi.org/10.1001/jamainternmed.2017. 6148. PubMed PMID: 29131896; PubMed Central PMCID: PMCPMC5838609.

34. Demiralp B, He F, Koenig L. Further Evidence on the System-Wide Effects of the Hospital Readmissions Reduction Program. Health Serv Res. 2018; 53(3): 1478-1497. Epub 2017/05/10. doi: https://doi.org/10.1111/1475-6773.12701. PubMed PMID: 28480598; PubMed Central PMCID: PMCPMC5980263.

35. Gai Y, Pachamanova D. Impact of the Medicare hospital readmissions reduction program on vulnerable populations. BMC Health Serv Res. 2019; 19(1):837. 31727168. https://doi.org/10.1186/s12913-019-4645-5. 
36. Press VG, Au DH, Bourbeau J, Dransfield MT, Gershon AS, Krishnan JA, et al. Reducing Chronic Obstructive Pulmonary Disease Hospital Readmissions. An Official American Thoracic Society Workshop Report. Ann Am Thoracic Soc. 2019; 16(2): 161-170. Epub 2019/02/02. doi: https://doi.org/10.1513/AnnalsA TS.201811-755WS. PubMed PMID: 30707066; PubMed Central PMCID: PMCP MC6812156.

37. Jennings JH, Thavarajah $K$, Mendez MP, Eichenhorn M, Kvale P, Yessayan L. Predischarge bundle for patients with acute exacerbations of COPD to reduce readmissions and ED visits: a randomized controlled trial. Chest. 2015;147(5):1227-34. Epub 2015/05/06. 25940250. https://doi.org/10.1378/ chest.14-1123.

38. Bhatt SP, Wells JM, lyer AS, Kirkpatrick DP, Parekh TM, Leach LT, et al. Results of a Medicare Bundled Payments for Care Improvement Initiative for Chronic Obstructive Pulmonary Disease Readmissions. Ann Am Thoracic Soc. 2017; 14(5): 643-648. Epub 2016/12/23. doi: https://doi.org/10.1513/A nnalsATS.201610-775BC. PubMed PMID: 28005410; PubMed Central PMCID: PMCPMC5802596.

39. Aboumatar H, Naqibuddin M, Chung S, Chaudhry H, Kim SW, Saunders J, et al. Effect of a Hospital-Initiated Program Combining Transitional Care and Long-term Self-management Support on Outcomes of Patients Hospitalized With Chronic Obstructive Pulmonary Disease: A Randomized Clinical Trial. JAMA. 2019; 322(14): 1371-1380. Epub 2019/10/09. doi: https://doi.org/10.1 001/jama.2019.11982. PubMed PMID: 31593271; PubMed Central PMCID: PMCPMC6784754.

40. Wadhera RK, Joynt Maddox KE, Kazi DS, Shen C, Yeh RW. Hospital revisits within 30 days after discharge for medical conditions targeted by the Hospital Readmissions Reduction Program in the United States: national retrospective analysis. BMJ (Clin Res ed). 2019; 366: 14563. Epub 2019/08/14. doi: https://doi.org/10.1136/bmj.14563. PubMed PMID: 31405902; PubMed Central PMCID: PMCPMC6689820.

41. Stein BD, Bautista A, Schumock GT, Lee TA, Charbeneau JT, Lauderdale DS, et al. The validity of International Classification of Diseases, Ninth Revision, Clinical Modification diagnosis codes for identifying patients hospitalized for COPD exacerbations. Chest. 2012; 141(1): 87-93. Epub 2011/07/16. doi: https://doi.org/10.1378/chest.11-0024. PubMed PMID: 21757568; PubMed Central PMCID: PMCPMC3251268.

42. US House of Representatives Document Repository. 21st Century Cures Act [cited 2020 November 2]. Available from: https://docs.house.gov/ billsthisweek/20161128/CPRT-114-HPRT-RU00-SAHR34.pdf.

43. McCarthy CP, Vaduganathan M, Patel KV, Lalani HS, Ayers C, Bhatt DL, et al. Association of the New Peer Group-Stratified Method With the Reclassification of Penalty Status in the Hospital Readmission Reduction Program. JAMA Netw Open. 2019; 2(4): e192987. Epub 2019/04/27. doi: https://doi.org/10.1001/jamanetworkopen.2019.2987. PubMed PMID: 31026033; PubMed Central PMCID: PMCPMC6487568.

44. Joynt Maddox KE, Reidhead M, Qi AC, Nerenz DR. Association of Stratification by Dual Enrollment Status With Financial Penalties in the Hospital Readmissions Reduction Program. JAMA internal medicine. 2019. Epub 2019/04/16. doi: https://doi.org/10.1001/jamainternmed.2019.0117. PubMed PMID: 30985863.

\section{Publisher's Note}

Springer Nature remains neutral with regard to jurisdictional claims in published maps and institutional affiliations.

Ready to submit your research? Choose BMC and benefit from:
- fast, convenient online submission
- thorough peer review by experienced researchers in your field
- rapid publication on acceptance
- support for research data, including large and complex data types
- gold Open Access which fosters wider collaboration and increased citations
- maximum visibility for your research: over 100M website views per year
At BMC, research is always in progress.
Learn more biomedcentral.com/submissions

